(C) [2008] IEEE. Reprinted, with permission, from [Aboura Khalid; Samali Bijan; Crews Keith; Li Jianchun, Stochastic Deterioration Processes for Bridge Lifetime Assessment, Proceedings of the Third International Conference on Broadband Communications, Information technology \& Biomedical Applications, 2008]. This material is posted here with permission of the IEEE. Such permission of the IEEE does not in any way imply IEEE endorsement of any of the University of Technology, Sydney's products or services. Internal or personal use of this material is permitted. However, permission to reprint/republish this material for advertising or promotional purposes or for creating new collective works for resale or redistribution must be obtained from the IEEE by writing to pubs-permissions@ieee.org. By choosing to view this document, you agree to all provisions of the copyright laws protecting it 


\section{Stochastic Deterioration Processes for Bridge Lifetime Assessment}

\author{
Khalid Aboura \\ University of Technology, Sydney \\ Centre for Built Infrastructure Research \\ 15 Broadway, Ultimo, NSW 2007, Australia \\ kaboura@eng.uts.edu.au \\ Keith Crews \\ University of Technology, Sydney \\ Centre for Built Infrastructure Research \\ 15 Broadway, Ultimo, NSW 2007, Australia \\ Keith.Crews@uts.edu.au
}

\author{
Bijan Samali \\ University of Technology, Sydney \\ Centre for Built Infrastructure Research \\ 15 Broadway, Ultimo, NSW 2007, Australia \\ Bijan@eng.uts.edu.au \\ Jianchun Li \\ University of Technology, Sydney \\ Centre for Built Infrastructure Research \\ 15 Broadway, Ultimo, NSW 2007, Australia \\ Jianchun.Li@uts.edu.au
}

\begin{abstract}
The Markov chain model can be found in the maintenance and repair problems since the early 60's, is introduced to the maintenance of road infrastructure in the 1980's, and is made to drive the current bridge maintenance optimization systems. While this model results into solvable programming problems and provides a solution, there are a number of criticisms associated with it. In this article, we highlight the shortfalls of the Markov model for bridge infrastructure lifetime assessment and promote the use of stochastic processes. We use examples from a study for the modeling of the condition of bridges that considers more than 15 years of data. We argue for the applicability of the gamma process and other stochastic processes.
\end{abstract}

\section{Introduction}

Bridge maintenance optimization was applied these past decades due to the large costs associated with the management of networks of ageing structures. In the United States, more than $70 \%$ of the bridges were built prior to 1935, and a large percentage of the United Kingdom's current bridge stock was built between the late 1950s and early 1970s. In the state of New South Wales, Australia, around $70 \%$ of the operating bridges were built before 1985, with a significant proportion before the 1940's. With the near completion of most of the road networks and the ageing of bridges, the emphasis shifted to the maintenance and rehabilitation of the existing infrastructure. A concerted effort was made in the 1970's after the collapse of several bridges in the United States in the late 60's [1]. A number of mandates by governments introduced standards and computerized maintenance optimization approaches. These software tools, known as Bridge Management Systems (BMS), consist of formal procedures and methods for gathering and analyzing bridge condition data. The purpose of a BMS is to predict conditions for bridge stocks and estimate maintenance funding. Pontis [2], one of the most widely used systems, was designed and developed at the request of the US Federal Highway Administration. A similar BMS is the BRIDGIT bridge management system [3]. A bridge in a BMS is represented by structural elements defined as a set of common bridge components. For each bridge, the conditions of these elements are assessed visually during periodic inspections, and reported into the BMS. The condition data at each inspection consist of the total quantity of the element being divided through a number of condition states; from the 'as good as new' condition to the most severe state of deterioration. This is a common practice for representing infrastructure condition data, starting with the discrete condition rating scale from 0 to 9 adopted by the U.S. Federal Highway Administration [4], and followed by the Pontis condition rating scale, and that of most other bridge inspection procedures. This process simplifies the inspections, but more importantly the modeling of the element/bridge/network condition and the maintenance optimization. The condition assessment and prediction is done by the application of a Markov chain model. The Markov chain model can be found in the maintenance and repair problems since the early 60's [5]. It is introduced to the maintenance of road infrastructure by Golabi et al. (1982) [6], and is made to drive the Pontis bridge management system. In the Markov model, the condition of a bridge ele- 
ment takes discrete states and the transitions from one state to the other are modeled with a Markov chain. While this approach has become standard, there have been a number of criticisms associated with it. In this article, we discuss the fundamentals and argue for the applicability of the gamma process and other stochastic processes for modeling structural deterioration.

\section{The Markov Chain model}

Markov model formulations are appealing to manage infrastructure because they provide a framework that accounts for the uncertainty and the optimal policies can be obtained by solving simple programming problems. A number of criticism points have been made against the usefulness of the model [1]; (i) bridge element performance is not addressed from a reliability viewpoint, (ii) the Markovian assumption does not take into account the history of the bridge deterioration, and (iii) bridge system performance is not generally addressed. Among these limitations, a most important one is the inability of the Markov model, by assumption, to capture the time effect of deterioration. It is clear to many practitioners that the deterioration rate tends generally to increase with time, and amount of deterioration. This observations conflicts with the stationarity assumption of the Markov model where the transition probabilities do not change with time. In the context of the estimation of the Markov model transition probabilities, Madanat, Mishalani \& Ibrahim (1995) [7] make a number of observations. They point to the fact that the methods used in estimating these probabilities are ad-hoc and suffer from important methodological limitations; (i) the change in condition from one inspection to the next is not modeled explicitly, failing to capture the structure of the deterioration process, (ii) consequently, the model fails to capture the inherent non-stationarity of the deterioration process, and (iii) the approach does not recognize the latent nature of deterioration. Since deterioration is an unobservable process, it is not the state of the observable condition that should be modeled, but rather the process that generates these conditions.

A practical difficulty with the estimation of the transition probabilities is the lack of data for some condition states. The severe conditions of elements of a bridge are rarely observed, due to the maintenance effort. Only the first states transition probabilities are estimated properly. Figure 1 shows the frequency of units of different elements in the possible condition states. These elements are taken from a network of bridges in the study we describe in the next section. Most of the element units are in state 2 when they deteriorate, that is leave the 'as good as new' or 'full' condition state 1 . While it depends on the element and on budget, many elements are brought back to the full condition state from state 2 . This implies that little data are available about transitions to more severe conditions. Pontis supplements the data with expert knowledge at the beginning of system implementation due to the scarcity of data at that stage. As more data becomes available, the probabilities are to be updated using a Bayesian method. This is a major shortfall, as many managers are reticent about the use of subjective assessment, particularly if there is little chance that enough data will appear to adjust the subjective input.

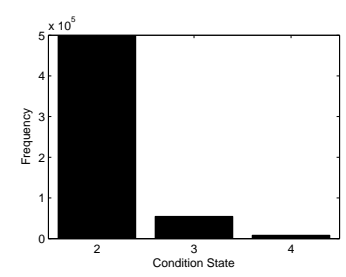

(a) Element example 1

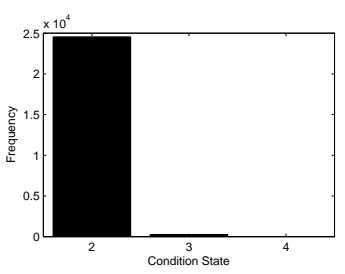

(b) Element example 2

\section{Figure 1. Condition state frequency}

The theoretical weakness of the Markov model is the assumption of stationarity. The model assumes that the time of transition from one state to another is distributed exponentially. This assumption allows the application of the Markov model by which an old unit of element and a newer one are equally likely to move to the next condition state [2]. This is a rigid structure imposed on the deterioration model. This weakness is acknowledged in most studies, and yet the Markov model has been adopted from the beginning and remains in most bridge management systems. It provides a solution for the estimation of future conditions and prescribes how to invest maintenance funding. However, a predictive solution relies on the validity of the underlying deterioration model. Scherer and Glagola (1994) [8], Wirahadikusumah et al. (2001) [9] and Morcous (2006) [10], among others have studied the validity of the state independence assumption of the Markov model. This assumption assumes that the future condition of a bridge element depends only on its present condition and not on its past condition, implying that bridge deterioration is a stationary process. While the simplifying assumption holds in some studies, it is not substantiated with a lot of evidence, the available condition data not providing adequate information about all possible condition transitions.

\section{The RTA Level-2 Inspection Data}

The goal in our study is to develop deterioration models for the assessment of future conditions of elements/bridges/networks in the state of New South Wales, Australia. The Roads and Traffic Authority (RTA) of the state of New South Wales, provided data that consist of inspection records conducted periodically on all bridges in 
their jurisdiction. The inspections are visual for most elements and the condition states are discrete. An element consists of a number of quantity units. Each unit is judged to be in one of $n$ states, $n=3,4$ or 5 , depending on the element. Condition state 1 represents the 'as good as new' condition, no-deterioration state, while condition states $2, \ldots, n$ mark increasing levels of deterioration. There are 4,945 structures and 66 elements considered in the study, for which over 230,000 inspection records exist. The records go back to 1989 , up to the present time. Among the recorded entries are $q$, the total quantity of the element, $q_{1}$ the quantity in condition state 1 on the day of that inspection for that particular element, $q_{2}$ the quantity in state 2 , to $q_{n}$ the quantity in condition state $\mathrm{n}$. The inspected quantities for each element are measured either in square meters $\left(\mathrm{m}^{2}\right)$ if it is a surface, in meters $(m)$ for some elements such as railing and joints, or units $(e a)$ for timber elements. The structures have on average about ten elements, with more than 30 in some cases. Each element was inspected approximately every two years. The elements are concrete elements, steel elements with lead based paint, steel elements with other protective treatment, timber elements, joints, bearings, railings and others. Some of the more common types are concrete pre-tensionned girder, concrete reinforced prestressed pile, concrete deck slab, concrete culverts, steel rolled beams/I girders with lead based paint protective coating, timber beam/cross girder, pourable/cork joint seal, elastomeric bearing, metal bridge railing and masonry/brick/reinforced earth.

Deterioration in elements of bridges is best measured as a continuous variable representing for example the percentage of degradation. Given the large surfaces and number of items to be inspected, it is hard to measure visually percentages. The condition data at each inspection therefore consists of the total quantity $q$ of the element being divided through judgement into $n$ states. $q=\sum_{i=1}^{n} q_{i}$ is satisfied in all inspection records. The percentage of undamaged quantity is $q_{1} / q \times 100 \%$. The corresponding proportion of deteriorated element is $q_{d}=\sum_{i=2}^{n} q_{i} / q, q_{d}=1-q_{1} / q$. If one was to ignore the degree of deterioration and consider only the proportion of damaged quantity, then $C=100 q_{1} / q$ provides a first level of information on the condition of the element. In our study, the condition data $\left(q_{1}, \ldots, q_{n}\right)$ of an element is converted to a univariate measure $C$, using the notion of 'Condition Index'. A number of condition indices were formulated. These indices can be related to the California bridge health index [11], a ranking system that takes values in $[0,100]$. The California Department of Transportation was involved in the development and implementation of Pontis. A condition index has two functions; (i) its use in a cost/benefit analysis where the condition history of a structure can be estimated with the inspection data of its elements, through the use of a weighted sum of the conditions of the elements, and (ii) its use in the study of deterioration by modeling the univariate measure in time.

\section{Estimating the Deterioration Rate}

In turning the vector of quantities $\left(q_{1}, \ldots, q_{n}\right)$ into a single value $C_{\text {elem }}(t)$, where $t$ is the time, the dimensionality of the problem is reduced and one can apply mathematically tractable models. This univariate quantity can be studied over time using a stochastic process. The element condition is a value between 0 and $100 \%, 100 \%$ being the 'as good as new' condition (no deterioration) state. The deterioration is defined as $Z=100-C,\left[C \equiv C_{\text {elem }}(t)\right]$. For the states where the deterioration has increased, and the difference provides information for the estimation of the stochastic process, the variables $Z_{1}, Z_{2}, Z_{3}, Z_{7}$ and $Z_{8}$ represent the deterioration. These states are:

Z1: The element condition is $100 \%$ and $100 \%$ at the previous inspection

Z2: The element condition is less than $100 \%$, but positive, while $100 \%$ at the previous inspection

Z3: The element condition is $0 \%$ while $100 \%$ at the previous inspection

Z7: The element condition is less than $100 \%$, but positive, and less than at the previous inspection where it was less than $100 \%$, but positive

Z8: The element condition is $0 \%$, while it was less than $100 \%$, but positive at the previous inspection

These variables are of relevance when studying the deterioration process, along with their inter-inspection times. The time at which a deterioration increase $(d Z$ in a time interval $d t$ ) occurs is important. The pairs $\{(d t, d Z)\}$, for $d Z \geq 0$, and the time at which $d t$ starts is information used to estimate the parameters of stochastic deterioration processes.

In order to study the distribution of the deterioration variables $Z_{2}$ and $d Z_{7}$ (the increase associated with $Z_{7}$ ), we needed data that occur at the same time. The interinspection times were adjusted so that they can be grouped together, making it possible to study the probabilistic behavior of deterioration as a random variable at different times. Judgement was also used in eliminating data for inspection time intervals that exceed 4 years. Beyond 4 years, the data start thinning. In the case of one element example, from the original 913 conditions, $C=100-Z_{2}$, 836 adjusted in time are plotted in Figure 2. Next, we used a least squares approach in fitting the data to a nonlinear deterioration curve $100-\mu t^{q}$, with parameters $\mu$ and $q$. Figure 2 displays the least squares results using (i) the condition means at times 1,2,3 and 4, (ii) all the adjusted data, and (iii) the 
actual data. $q$ was found to be $2.7,2.4$ and 2.4 respectively, and $\mu=0.29,0.404,0.408$, showing that the adjustment to the data was minimal. The time pattern of the deterioration of the element fits the behavior of a stochastic process such as the gamma process.

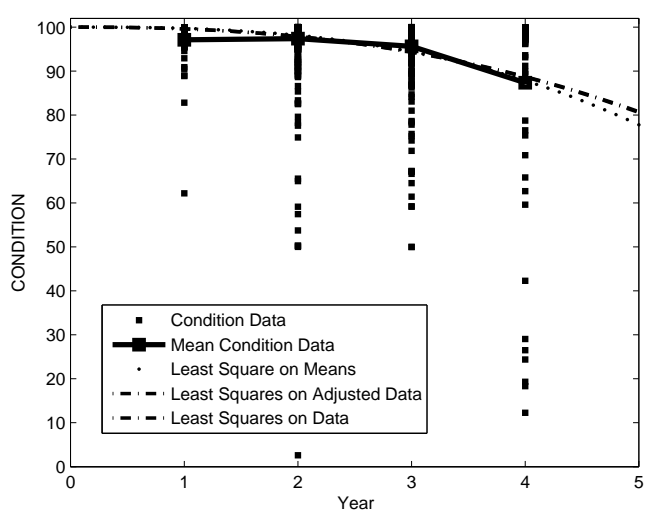

Figure 2. Condition of a concrete element

The distributions of $Z_{2}$ at different points in time charcaterize the stochastic process. In the case of most of our elements, the probability distribution showed a good fit to the gamma distribution (Fig. 3). This concords with the properties of the gamma deterioration process, where the distributions of incremental deteriorations are gamma distributed [12]. This was observed for the concrete and steel elements, as well as railing and joints and some timber elements. The lognormal was also found to be a good fit (Figure 4) and in most cases with enough data, a better fit. In

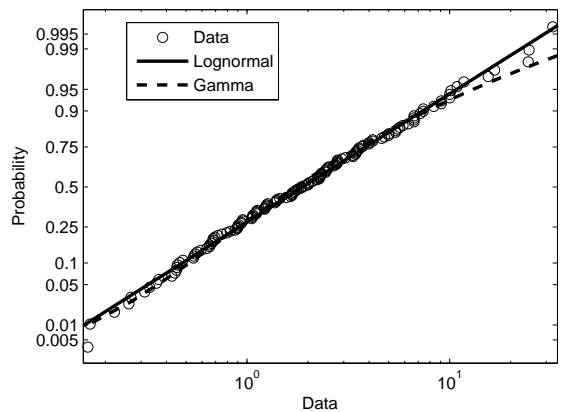

Figure 3. Probability distribution fits

the gamma process, not only is the distribution a gamma distribution when measured from the start of time, but any incremental distribution is also a gamma distribution. That is, if taking an interval in time and measuring the difference, increase, in the quantity of interest, the distribution of such an increase is also a gamma distribution. In addition, the increases in non-overlapping time intervals are

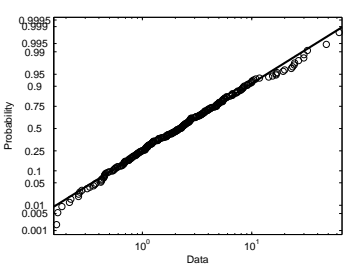

(a) Concrete Element

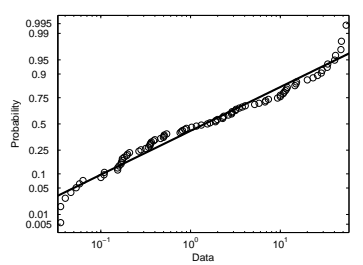

(b) Steel Element

\section{Figure 4. Lognormal probability fit}

independents. This makes for a number of assumptions to be checked and is the subject of ongoing work in our study. However, we have already observed, whenever $d Z 7$ data are abundant enough, that these assumptions can be accepted. The data of this study show the behavior of a stochastic process with selected probability distributions. At this point, the data have not been stratified according to influencing factors such as traffic load, age of bridge and location, except in some examples. The observations of distribution fit held in those cases. The observation also held when the data were stratified into different structure (bridge) classes, according to exact and major components similarity.

For the purpose of building a statistical model, these observations promote the use of the gamma process with mean deterioration function $\mu t^{q}$ that includes the stationarity case of $q=1$.

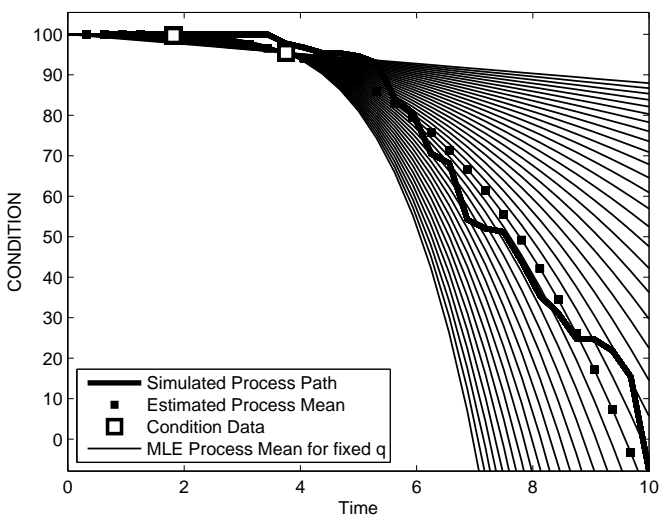

Figure 5. Gamma process estimation, $\hat{\mathbf{q}}=\mathbf{3 . 3}$

The gamma process is defined by van Noortwijk (2007) [12] in the context of the deterioration of structures. To illustrate the estimation procedure, we apply it to a concrete element on two different bridges and observe a rate $\hat{q}=$ 3.3 (Fig. 5). Two data points are used; $(15.81,99.69)$ and $(17.75,95.46)$ with the renewal at year 13.92 since the start of the database. This example was chosen for illustrative purposes. In many cases, the bridge elements showed lower rates. 


\section{The Gamma Process}

The gamma process can capture the temporal variability of degradation. The argument is made in a series of papers by Pandey and Noortwijk (2004) [13], Noortwijk, Kallen and Pandey (2005) [14] and Pandey, Yuan and Noortwijk (2007) [15]. The gamma process can be found in its modern application to structures in the late 90 s by van Noortwijk (1998) [16] and van Noortwijk and Klatter (1999) [17]. The idea of the use of a gamma process can be found earlier in the Netherlands where generalized gamma processes were used to model decision problems for optimizing maintenance of the sea-bed protection of the Eastern-Scheldt barrier, berm breakwaters, and dykes (van Noortwijk \& van Gelder, 1996) [18]. Empirical studies showed that the expected deterioration in some cases followed the power law $a t^{b}$, where $t$ is the time. This function of time is incorporated into the gamma process and used to model structural deterioration. The advantage of the gamma process is recognized and applied in many structural studies [19, 20, 21]. van Noortwijk (2007) [12] provides a comprehensive overview of the use of the gamma process in the maintenance of structures. In the context of structural deterioration, the gamma process is defined as follows: Let $v(t)$ be a non-decreasing, right continuous, real-valued function for $t \geq 0$, with $v(0)=0$. The gamma process with shape function $v(t)>0$ and scale parameter $u>0$ is a continuous-time stochastic process $\{Z(t), t \geq 0\}$ with the following properties:

1. $Z(0)=0$ with probability 1

2. $Z(\tau)-Z(t) \sim G(v(\tau)-v(t), u)$

3. $Z(t)$ has independent increments

where $G(z \mid v, u)=u^{v} z^{v-1} e^{-u z} / \Gamma(v)$ is the gamma probability density function defined for $z \in(0, \infty)$. The process can be parameterized. Letting $v(t)=\mu^{2} t^{q} / \sigma^{2}$ and $u=\mu / \sigma^{2}$, the mean and variance of the deterioration $Z(t)$ are:

$$
E(Z(t))=\mu t^{q} \text { and } V(Z(t))=\sigma^{2} t^{q} .
$$

Given a set of observations of the deterioration process $Z(t),\left\{z_{i}\right\}_{i=1}^{n}$ for times $\left\{t_{i}\right\}_{i=1}^{n}$, the maximization of the likelihood function provides estimates of the three parameters $\mu, \sigma$ and $q$. This involves the search, $\mathrm{q}$ fixed, for the zero of a function, where $\hat{\sigma}$ is solution of

$$
\sum_{i=1}^{n} w_{i}\left\{\Psi\left(\frac{\hat{\hat{\mu}}^{2}}{\sigma^{2}} w_{i}\right)-\log \delta_{i}\right\}=t_{n}^{q} \log \left(\frac{x_{n}}{t_{n}^{q} \sigma^{2}}\right)
$$

where $\delta_{i}=z_{i}-z_{i-1}, i=1, \ldots, n, z_{0}=0$, and $w_{i}=$ $t_{i}^{q}-t_{i-1}^{q}, i=1, \ldots, n$, with $t_{0}=0$, and $\hat{\hat{\mu}}=z_{n} / t_{n}^{q} . \Psi$ function is the Digamma function.
The data for each individual bridge aren't enough to estimate the parameters $\mu, \sigma$ and $q$ properly. Often, the path $\left\{z_{i}\right\}_{i=1}^{n}$ for times $\left\{t_{i}\right\}_{i=1}^{n}$ does not extend beyond $n=1$ or 2 , before the element is brought back to the full condition state $(Z=0)$. One way around this problem, and the advantage of using the gamma process, is to aggregate the data by dividing the bridges into similarity classes. Bridges often can be grouped through the identification of some major elements. Then within the classes of similar bridges, a second stratification occurs according to influencing factors, such as traffic load, age, region and environmental stress. The estimation of the parameters within the class structures results in better assessment and prediction. Due to the independent increments property and with the assumption of independence between elements on different structures, the likelihood function can be written and maximized to estimate the parameters $\mu, \sigma$ and $q$ [21]. In the special case where only the first deteriorations, observed at the first inspection after a renewal, are available, then the solution is similar to that seen above, but the search for $\hat{\sigma}$ involves the equation

$$
\sum_{i=1}^{n} w_{i}\left\{\Psi\left(\frac{\hat{\hat{\mu}}^{2}}{\sigma^{2}} w_{i}\right)-\log \delta_{i}\right\}=\left(\sum_{i=1}^{n} w_{i}\right) \log \left(\frac{\hat{\hat{\mu}}}{\sigma^{2}}\right)
$$

where $z_{i}, i=1, \ldots, m$, this time is the deterioration of the $i^{t h}$ element in the class of structures, $\delta_{i}=z_{i}$ and $w_{i}=t_{i}^{q}$, and $\hat{\hat{\mu}}=\sum_{i=1}^{n} z_{i} / \sum_{i=1}^{n} t_{i}^{q}$.

\section{Conclusion}

Structural deterioration assessment and condition prediction of bridges is a subject of interest that has far reaching consequences, both in terms of public safety and state budgeting. We discussed the theoretical foundations of the model most used for assessing structural deterioration in bridge elements. We propose a modern view using the gamma process that has already proved successful in structural deterioration assessment. We experimented with the process using simulation, estimating the parameters with the maximum likelihood approach. With few data points, the model captures the deterioration process efficiently. We also observed in our study a distribution for the deterioration other than the gamma distribution. This leads to the application or development of other stochastic deterioration processes.

\section{Acknowledgement}

The authors wish to acknowledge the financial support provided by the Roads and Traffic Authority of N.S.W. for this research project. 


\section{References}

[1] D.M. Frangopol, J.S. Kong and E.S. Gharaibeh, "Reliability-based life-cycle management of highway bridges", Journal of Computing in Civil Engineering, ASCE 15(1), 2001, pp. 27-34.

[2] K. Golabi and R. Shepard, "Pontis: A system for maintenance optimization and improvement of US bridge network", Interfaces 27(1), 1997, pp. 71-88.

[3] H. Hawk and E.P. Small, "The BRIDGIT bridge managment system", Structural Engineering International, 8(4), 1998, pp. 309-314.

[4] Federal Highway Administration, "Recording and coding guide for structure inventory and appraisal of the nation's bridges", U.S. Department of Transportation, Washington, D.C., U.S.A, 1979.

[5] M. Klein, "Inspection-maintenance-replacement schedules under Markovian deterioration”, Management Science 9(1), 1962, pp. 25-32.

[6] K. Golabi, R.B. Kulkarni and G.B. Way, "A Statewide Pavement Management System", Interfaces 12(6), 1982, pp. 5-21.

[7] S. Madanat, Mishalani, R. and W.H. Wan Ibrahim, "Estimation of infrastructure transition probabilities from condition rating data", Journal of Infrastructure Systems 1(2), 1995, pp. 120-125.

[8] W.T Scherer and D.M. Glagola, "Markovian models for bridge maintenance management", Journal of Transportation Engineering, 120(1), 1994, pp. 37-51.

[9] R. Wirahadikusumah, D. Abraham and T. Iseley, "Challenging issues in modeling deterioration of combined sewers", Journal of Infrastructure Systems 7(2), 2001, pp. 77-84.

[10] G. Morcous, "Performance Prediction of Bridge Deck Systems Using Markov Chains", Journal of Performance of Constructed Facilities 20(2), 2006, pp. 146155.

[11] R.W. Shepard and M.B. Johnson, "California Bridge Health Index", IBMC-005, California Department of Transportation, International Bridge Management Conference, Denver, Colorado, Preprints, Volume II, K1, 1999.

[12] J.M. van Noortwijk, "A survey of the application of gamma processes in maintenance", Reliability Engineering and System Safety, 2007.
[13] M.D. Pandey and J.M. van Noortwijk, "Gamma process model for time-dependent structural reliability analysis", In E. Watanabe, D.M. Frangopol, and T. Utsonomiya, editors, Bridge Maintenance, Safety, Management and Cost, Proceedings of the Second International Conference on Bridge Maintenance, Safety and Management (IABMAS), Kyoto, Japan, 18-22 October 2004. London: Taylor \& Francis Group.

[14] J.M. van Noortwijk, M.J. Kallen and M.D. Pandey, "Gamma processes for time-dependent reliability of structures", In K. Kolowrocki, editor, Advances in Safety and Reliability, Proceedings of ESREL 2005 - European Safety and Reliability Conference 2005, Tri City (Gdynia-Sopot-Gdansk), Poland, 27-30 June 2005, pp. 1457-1464. London: Taylor \& Francis Group.

[15] M.D. Pandey, X.-X. Yuan and J.M. van Noortwijk, "The influence of temporal uncertainty of deterioration on life-cycle management of structures", Structure and Infrastructure Engineering, 2007, pp. 1-12.

[16] J.M. van Noortwijk, "Optimal Replacement Decisions for Structures under Stochastic Deterioration", in Andrzej S. Nowak, editor, Proceedings of the Eighth IFIP WG 7.5 Working Conference on Reliability and Optimization of Structural Systems, Krakow, Poland, 1998, pp. 273-280, Ann Arbor: University of Michigan.

[17] J.M. van Noortwijk and H.E. Klatter, " Optimal inspection decisions for the block mats of the EasternScheldt barrier", Reliability Engineering and System Safety, 65, 1999, pp. 203-211.

[18] J.M. van Noortwijk and P.H.A.J.M. van Gelder, "Optimal maintenance decisions for berm breakwaters", Structural Safety, 18(4), 1996, pp. 293-309.

[19] F.A. Buijs, J.W. Hall, J.M. van Noortwijk and P.B. Sayers, "Time-dependent reliability analysis of flood defences using gamma processes", In: Augusti G, Schueller GI, Ciampoli M, editors. Safety and reliability of engineering systems and structures; pp. 22092216. Rotterdam: Millpress; 2005.

[20] J.M. van Noortwijk, J.A.M. van der Weide, M.J. Kallen, M.J. and M.D Pandey, "Gamma processes and peaks-over-threshold distributions for time-dependent reliability", Reliability Engineering and System Safety 92, 2007, pp. 1651-1658.

[21] R.P. Nicolai, R. Dekker and J.M. van Noortwijk, "A comparison of models for measurable deterioration: An application to coatings on steel structures", Reliability Engineering and System Safety 92, 2007, pp. 16351650 . 